\title{
The Clinical Case of Keratopathy After Phacoemulsification
}

\author{
Svirin AV, Rabanova MG and Gribkova IV* \\ Russian National Research Medical University, Russia \\ *Corresponding author: IV Gribkova, Russian National Research Medical University, Ostrovityanova str., Russian Federation, Moscow, Russia
}

Submission: 眥July 02, 2018; Published: 制 September 12, 2018

\section{Introduction}

Pseudophakia bullous keratopathy (PBK) and a phakic bullous keratopathy $(\mathrm{ABK})$ refer to the development of irreversible corneal edema as a complication of cataract surgery. The history of PBK parallels the history of the development of the intraocular lens. As surgical techniques and lens design have improved, the incidence of this complication has decreased dramatically. However, it still represents an important cause of visual disability following routine and complicated cataract surgery. First, in a postoperative period edema effects cornea of those patients who undergo before keratorefractive surgery - such as radial keratotomy, or those who has prior corneal opacity after traumas or surgery.

Corneal transparency is, in a large part, dependent on the ability of the cornea to remain in a dehydrated state. It is affected by several interdependent factors:

A. The size of intraoperation discussion

B. Volume of ultrasound exposure

C. Model of used IOL

D. Toxic or aggressive effects of pre-or intraoperative assessment, or postoperative therapy.

The epithelium and the endothelium are both semipermeable membranes that create a barrier to the flow of water and other electrolytes into the cornea. Evaporation from the corneal tear film results in slightly hypertonic tears that tend to draw fluid out of the cornea. Intraocular pressure tends to drive fluid into the cornea. Osmotic forces and the electrolyte balance within the corneal stroma also tend to draw water into the cornea. However, the most important influence on corneal detergence is the presence of an active metabolic pump in the endothelium.

According to some studies, it was found that in cases with standard IOL models, a reduction in the diameter of the incision significantly reduces the postoperative trauma, in particular, the results of the operation with basic access of 3.0 and $2.75 \mathrm{~mm}$ were worse than in the $2.2 \mathrm{~mm}$ section.
In cases of mature cataract exposure time and ultrasound power is much greater than if there is a slight opacity in the lens. And despite the protective properties of viscoelastic, the surrounding tissues suffer from the damaging effects of ultrasound. The risk of developing bullous keratopathy increases with the use of anterior chamber IOLs, iris-clip lenses, in compare to posterior chamber IOL models.

As for toxic effects on the cornea, it is provided by irrigation solutions, anesthetics, antibiotics injected into the eye during surgery, viscoelastic and lens mass, as well as eye drops, and ointments trapped inside the eye due poor hermetic sealing of the incisions.

\section{Case Presentation}

The patient, a 30- year- old woman, complained of Poor vision, discomfort, Pain, Foreign body sensation.

In 1993 at the age of 6 years she has penetrate her right eye with a sharp object (scissors), with the further development of posttraumatic cataract. At the first stage was performed primary surgical treatment on her cornea, then extraction of traumatic cataract. In 2006, the implantation of a monotonal IOL was performed.

The patient never used obstacles or contact correction. Low objective vision of the right eye persisted throughout her life, medical local therapy and other types of treatment were not conducted. Since 2006, she hasn't visited ophthalmologist.

A standard ophthalmological examination was performed, as well as pachymetry, an optical coherent tomography of the anterior segment, ultrasound scans, a Schirmer test, a fluorescein test [1-7].

Visual acuity VIS OD=20\200

Vis OS $=20 / 32$ sph -1.5 cyl -0.37 ax $65=20 / 20$

IOP OD-palpatory norm.

IOP OS-according to pneumotonometry data was $17 \mathrm{~mm} \mathrm{Hg}$ 


\section{Slit lamp examination.}

OU inflammation of the eyelids edge, dysfunction of the meibomian glands, the meibomian glands ducts stain with fluorescein, the conjunctiva has mild irrigation, local folliculitis Figure 1. Also, we observed external strabismus Figure 2.

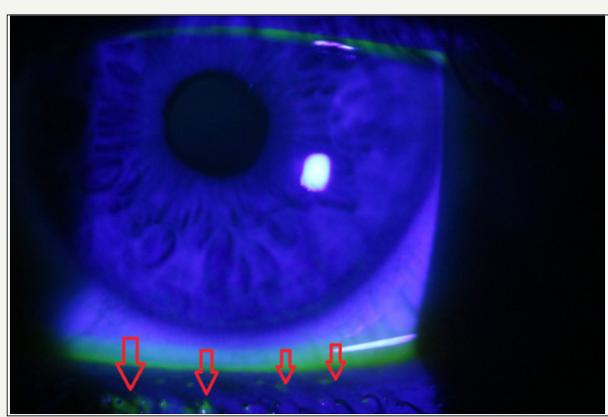

Figure 1: OS Fluorescine test 1.

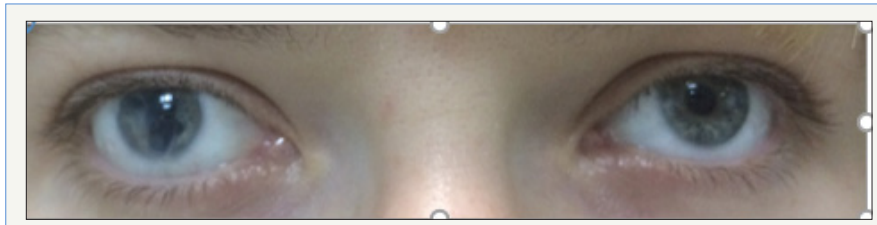

Figure 2: Strabismus 1.

OD on the cornea - posttraumatic scar in the lower part reaching the optical center. Slit lamp examination reveal obvious overall thickening and cloudiness of the central and peripheral cornea, in the temporal half - lining and bends formed by the basal membrane. The anterior chamber was deep, the iris has local atrophy segments, the pigment leaf was turned into the anterior chamber. The pupil is irregular in shape, stretched towards the atrophy part of the iris, photoreaction was absent. IOL located in the back chamber, in correct position Figure 3.

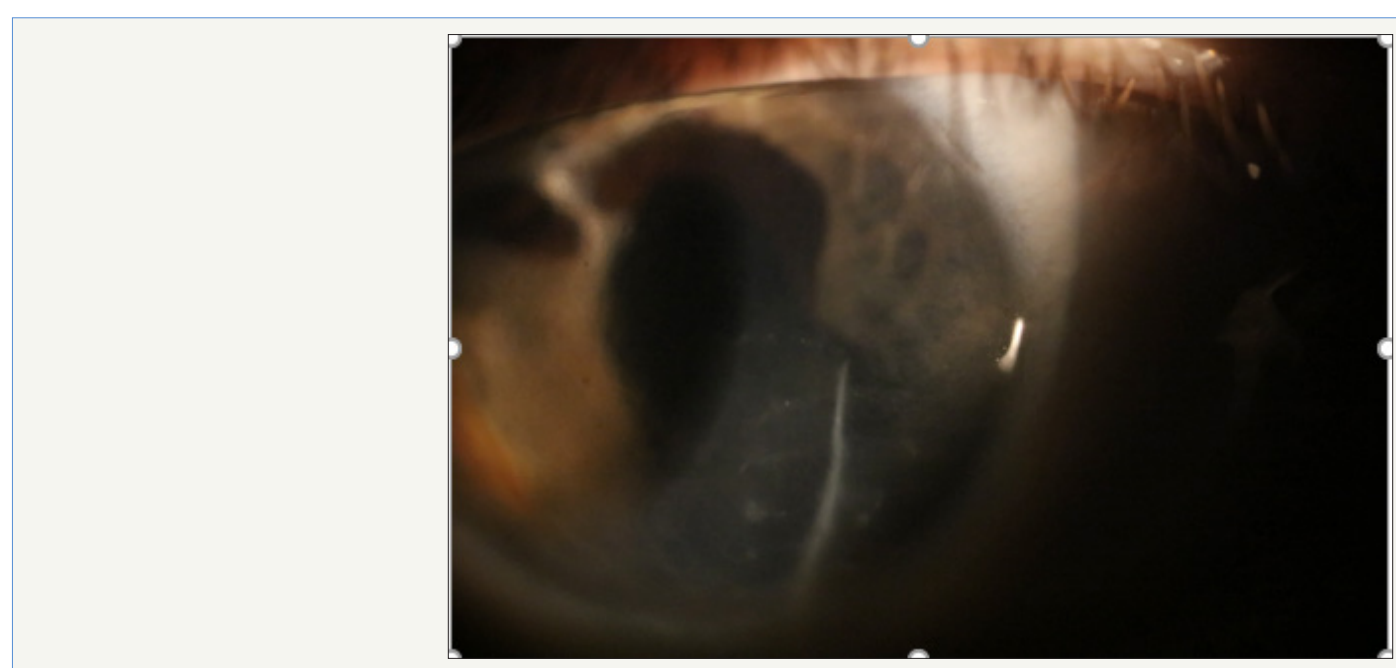

Figure 3: OD - slit lamp exam.

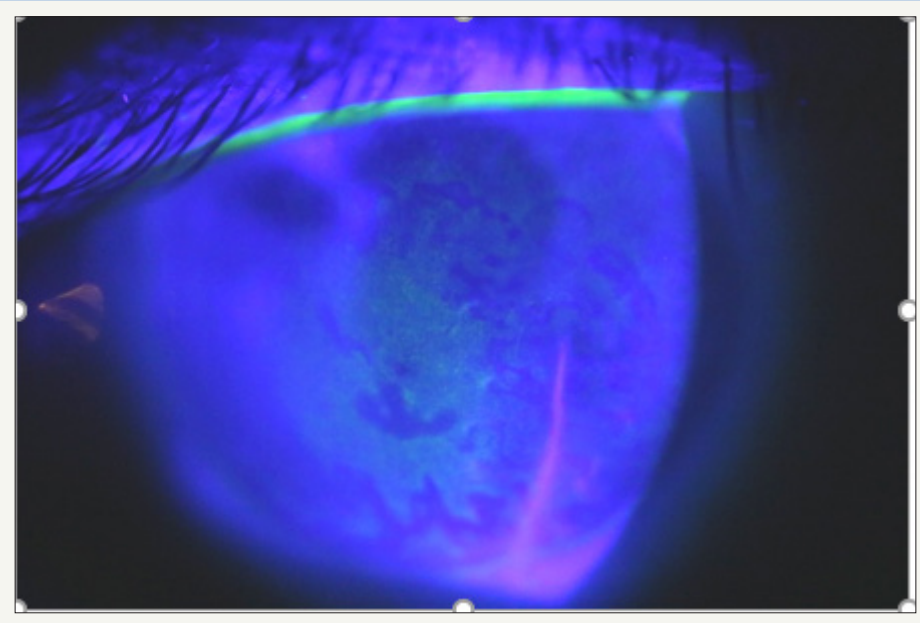

Figure 4: OD - fluorescine test.

In the center of cornea, we mentioned the area of thickening of the epithelium with borders like geographical map, weakly stained with fluorescein. adjacent areas were well stained with fluorescein and appear brighter the reflex from the fundus was pale pink, the details were not visualized Figure 4. According to the optical coherence tomography of the anterior segment we found a hypoechoic formation with distinct contours in the corneal stroma (intrastromal cyst). Also, there was visible the deformation of the iris in the temporal half and the areas of its contact with the IOL. OS state of optical part and the fundus - with no pathology Figure 5. 


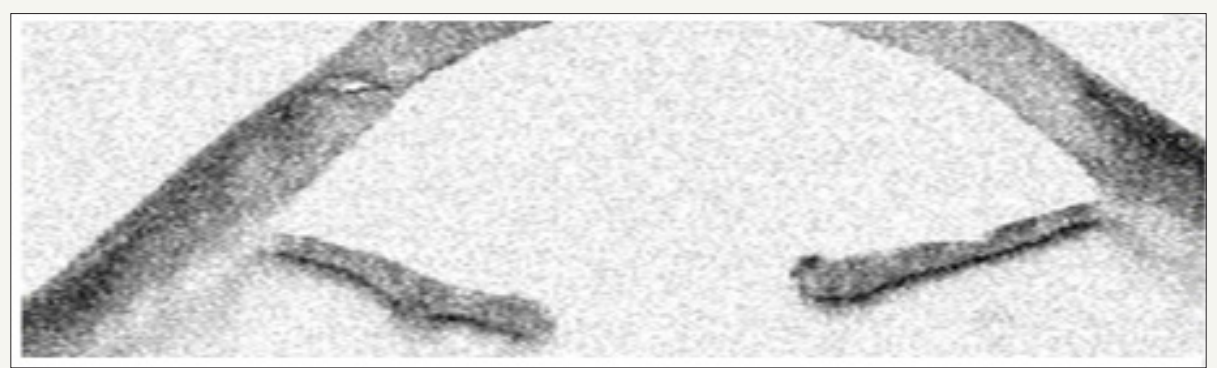

Figure 5: OD -OCT

According to the pachymetry, there was diffuse increase in the thickness of the cornea and in average it is $752 \mu \mathrm{m}$ in the center Figure 6. The Schirmer test of OD was $10 \mathrm{~mm}$, OS $10 \mathrm{~mm}$. Tear film burst time was OD 9sec OS 8sec. Ultrasound investigation OD - showed that in the posterior parts of the vitreous body was dense haze, with fuzzy contours, fixed to the retina, mainly in the lower parts Figure 7.

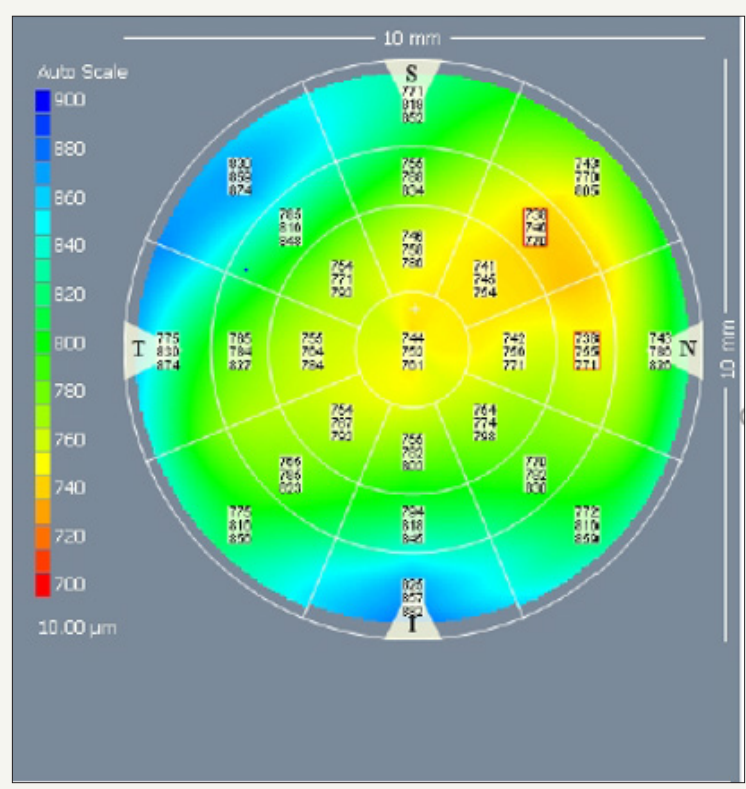

Figure 6: OD Pachymetry.
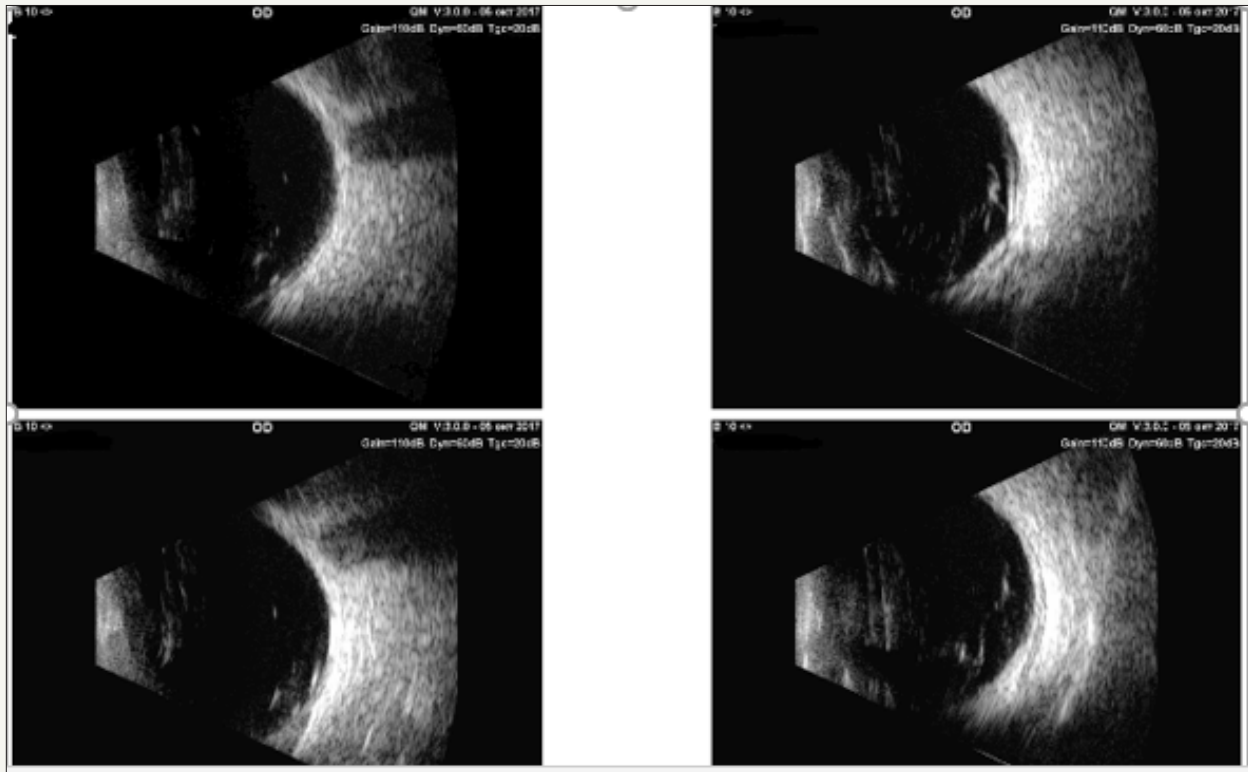

Figure 7: OD Bscan. 
Diagnosis - Right eye: posttraumatic scar of the cornea, bullous keratopathy, pseudophakia, destruction of the vitreous body, divergent strabismus. Amblyopia of high degree. Left eye myopia of weak degree. Both eyes: Dry-eye type I degree. Chronic blepharoconjunctivitis.

\section{Discussion}

The patient was prescribed complex instillation therapy contained eye drops and eye gel. As the main therapy in the treatment of dry eye syndrome, an artificial tear drops were administered in a non-conserved form, because of side effects of preservatives, which have a negative effect on the dynamics of the disease, such as:

a. Destabilize the tear film, destroys its lipid component, accelerating evaporation

\section{b. Destroy intercellular contacts \\ c. Have a cytotoxic effect \\ d. Cause allergic reactions.}

For daily use, was prescribed an eyelid hygiene special eye gel for dissolving and removing epithelial remains from the skin, secretions of sebaceous, meibomian glands and crusts. As well as sterile wipes, also containing no preservatives, to treat eyelids with an anti-inflammatory purpose, to improve microcirculation and restore conjunctival tissue.

Also, radioprotectors were prescribed: DE panthenol in the form of an eye gel to stimulate tissue regeneration, reduce the activity of the process, reduce the corneal edema. At the same time, NSAIDs were prescribed as eye drops to inhibit the chronic inflammatory process in the anterior part of the eye.

The patient was observed for 3 months, the examinations were carried out every 2 weeks. The patient daily performed a procedure of hygienic cleaning of the eyelids with wipes, contained botanical extracts and eye gel with Poloxamer 188 - for the entire observation period.

To repair the conjunctival cavity, an antiseptic of a broad spectrum -Picloxydine $0.05 \%$ - was prescribed.

artificial tears medication, Tregalose 3\% was prescribed 2-3 times a day - for the entire period of observation in both eyes, Piloxidin 0.05\% 3 times a day - 3 weeks, Dexpanthenol 4-5 times a day - in the right eye - on the entire observation period, Indomethacin 3 times a day - for 3 weeks in the right eye.

\section{Conclusion}

There was a positive dynamic - a decrease in symptoms of SSH, an improvement in the scores of the Schirmer test and VRSP, a decrease in edema and thickness of the cornea, a reduction in the boundaries of the dystrophic focus on the cornea.

This clinical example demonstrates the need to carefully consider the factors of pre- and postoperative management of patients at high risk of developing of bullous keratopathy after phacoemulsification. In such cases, a more careful approach to the choice of operational techniques should be applied: reduction of the incision diameter, reduction of the time of ultrasound exposure, application of preservative-free forms of instillation therapy in the pre- and postoperative period and in the long term, taking into account the compromised condition of the entire anterior segment of the eye and especially of the cornea. Such a category of patients' needs regular ophthalmologist's check-up for timely detection of pathology and correction of corneal abnormalities to reduce the rates of vision impairment.

\section{References}

1. Claesson M, Armitage WJ, Stenevi U (2009) Corneal oedema after cataract surgery: predisposing factors and corneal graft outcome. Acta Ophthalmol 87(2): 154-159.

2. Smolin G, Thoft, RA, Dohlman CH (1994) Endothelial function. The Cornea: Scientific Foundations and Clinical Practice. ( $3^{\text {rd }}$ edn), Lippincott William \& Wilkins, USA, pp. 635-643.

3. Stark WJ, Worthen DM, Holladay JT, Bath PE, Jacobs ME, et al. (1983) The FDA report on intraocular lenses. Ophthalmology 90(4): 311-317.

4. Taylor DM, Atlas BF, Romanchuk KG, Stern AL (1983) Pseudophakic bullous keratopathy. Ophthalmology 90(1): 19-24.

5. Waring GO (1989) The 50-year epidemic of pseudophakic corneal edema. Arch Ophthalmol 107(5): 657-659.

6. (1987) Closed-loop anterior chamber lenses. Arch Ophthalmol 105(1): $19-21$.

7. Hagan JC (1987) A clinical review of the IOLAB Azar model 91Z flexible anterior chamber intraocular lens. Ophthalmic Surg 18(4): 258-261.
Creative Commons Attribution 4.0 International License

For possible submissions Click Here

\section{Submit Article}

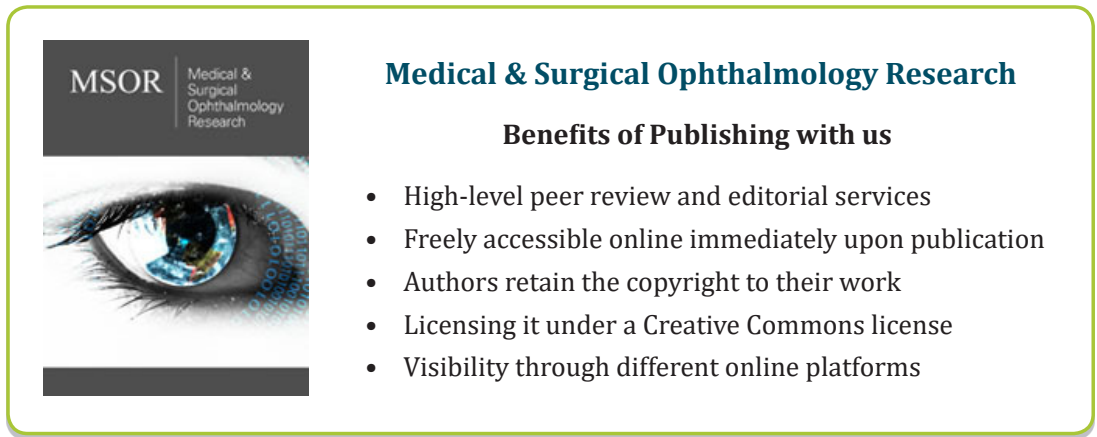

